\title{
Perturbation based stochastic isogeometric analysis for bending of functionally graded plates with the randomness of elastic modulus
}

\author{
Hien Duy Ta ${ }^{a *}$ (D), Phu-Cuong Nguyen ${ }^{b}$ \\ ${ }^{a}$ Department of Civil Engineering, University of Transport and Communications, Hanoi, Vietnam. Email: tdhiengt@gmail.com, \\ tdhien@utc.edu.vn \\ ${ }^{b}$ Department of Structural Engineering, Ho Chi Minh City Open University, 97 Vo Van Tan, Ho Chi Minh City, Vietnam. Email: \\ cuong.pn@ou.edu.vn, henycuong@gmail.com
}

* Corresponding author

https://doi.org/10.1590/1679-78256066

\begin{abstract}
We propose, in this paper, stochastic isogeometric analysis (SIGA) is a type of non-statistic approach in which combines the perturbation technique with the standard isogeometric analysis, in particular for static behavior of functionally graded plates with the uncertain elastic modulus. We assume that the spatial random variation of elastic modulus can be modeled as a two dimensional Gaussian random field in the plane of the plate. The random field is discretized to set of random variables using the integration point method. The system equations of SIGA are created using the NURBS functions for approximation displacement fields in conjunction with the first-order and second-order perturbation expansions of random fields, stiffness matrix, displacement fields. Besides the non-statistic approach, Monte Carlo simulation is presented for validation. The accuracy and appropriateness of the non-statistic approach are demonstrated via comparisons of the present results with those given by the stochastic finite element method in the literature and by the Monte Carlo analysis as well. The numerical examples are employed to investigate the effect of the randomness of elastic modulus and system parameters on the first and second statistical moments of displacement.
\end{abstract}

\section{Keywords}

SIGA, FGM plates, random field, elastic modulus, perturbation technique.

\section{Graphical Abstract}

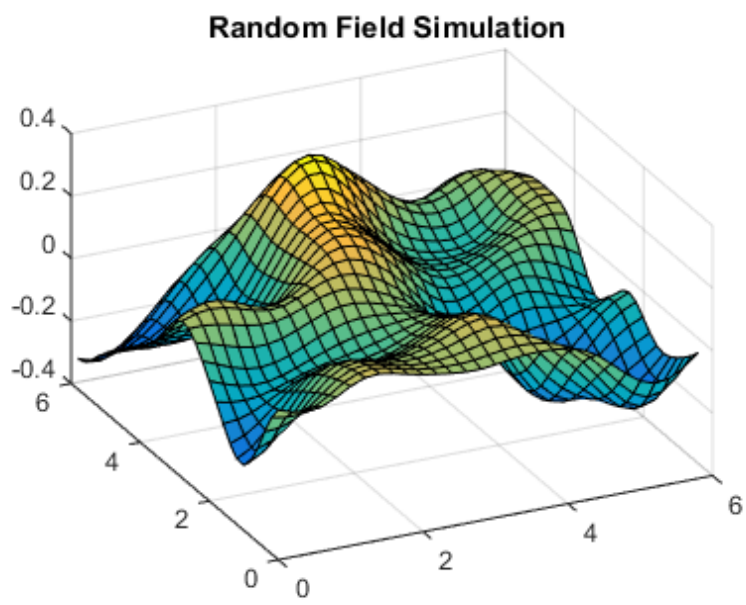




\section{INTRODUCTION}

Functionally graded material is a type of composite material which material components vary continuously along the thickness. There are also similar functionally graded materials in nature such as bones and bamboo trees, which have natural functional grading. Typical functionally graded materials are made from ceramic and metal to improve heatproof properties and achieve high toughness (Koizumi 1997; Shiota and Miyamoto 1997; Miyamoto et al. 1999). Many research was conducted to investigate the behavior of beam, plate and shell made of functionally graded materials in the literature (Ha and Khue 2019; Hafed and Zenkour 2019; Phuc 2019; Vuong and Duc 2019)

To compute structural behavior, structural systems are commonly idealized as a mathematical model in which system parameters such as the geometry of structures, material properties, and loading are related to the response of structures such as displacements, strains, and stresses. Researches of computational structural mechanics are divided into two major areas: deterministic analysis and nondeterministic analysis. In general, computational analyses in practice do not deal with the problems involving uncertainties in structural parameters. That is, they ignore the random heterogeneity of materials (e.g., soil, concrete, composites) and loading (e.g., vehicle, wind, wave) and favor using deterministic models with average or extreme values so that it leads to a crude representation of physical behavior with an unignorable error. The advanced structural models, in fact, need to encompass more detailed information on the involved system parameters. Accordingly, the analysis of structures needs to take into account uncertain parameters of structures and to estimate their effect on the responses.

Probabilistic computation on structures with uncertain parameters falls basically into two main categories: methods using a statistical approach and methods using a nonstatistical approach. The statistical approach involves the numerical generation of random parameters and statistical estimation of response due to the random parameters. This process is called Monte Carlo simulation (MCS). Among the nonstatistical approaches, most of the reports in the literature are associated with the stochastic finite element method (SFEM). There are a variety of SFEM schemes depending on how the uncertain parameters are dealt with. For example, Liu et al. (1986) suggested an SFEM called the "probabilistic finite element method" using the interpolation method for computing random fields. Stochastic finite element analysis (Takada 1990; Deodatis 1991; Wall and Deodatis 1994; Noh 2004; Hien 2020) has used the weighted integral of random fields on the finite element area as random variables; Spectral SFEM used Karhunen-Loève expansion series Ghanem and Spanos (1991) or homogeneous chaos expansion method Galal et al. (2008) for the representation of random fields.

Several studies have adopted the SFEM for structural analysis considering random system properties. The SFEM using a weighted integral approach Noh (2011) investigated the response variability of the bending of plate structures. Singh et al. (2008) studied the bending of a laminated composite plate considering uncertain system properties. Zhu and Wu (1991) developed a scheme for both distinct and repeated eigenvalue analysis using SFEM based on a local average technique. Graham and Deodatis (2001) used SFEM to investigate the variability of response of displacements and eigenvalues of structures with multi-uncertain parameters. Shang and Yun (2013) used ABAQUS combined with Karhunen-Loève expansion to simulate the stochastic response of structures under material uncertainties. Jun et al. (2014) analyzed the response of composite beam under stochastic excitations. Kaminski (2001) investigated the response variability of strain and stress tensors in the beam with random material and geometrical parameters. Cavdar et al. Cavdar et al. (2008) applied the perturbation based stochastic finite element method for predicting the response of three-dimension composite frames under earthquake forces. Real et al. (2017) proposed the new approach using CraigBampton method to model the uncertain dynamic systems.

Some researchers investigated the static, dynamics and buckling problems of FGM plates with random variables. Talha and Singh (2014) applied a perturbation technique to the finite element method for buckling of FGM plates considering uncertain material properties in thermal environments. Chakraborty and Rahman (2008) studied stochastic multiscale models for fracture analysis of functionally graded materials.

Recently, besides the SFEM, some researchers suggested a mesh-free method in investigating structures with uncertainties. For example, Rahman and Rao (2001) extended the element-free Galerkin method to develop a stochastic meshless method for the linear elasticity problem. Arun et al. (2010) combined Karhunen-Loève expansion with an element-free Galerkin method for the elastoplastic problem. Kim and Inoue (2004) developed the spectral stochastic element-free Galerkin method adopting Karhunen-Loève expansion and Polynomial Chaos series for the linear elastic problems involving random material properties. Hosseini and Shahabian (2014) investigated the stochastic elastic wave propagation in a thick hollow cylinder using a stochastic hybrid mesh-free method. 
In recent years, isogeometric analysis (IGA) using nonuniform rational B-splines (NURBS) was proposed by Hughes et al. (2005). There have been many studies on the IGA for structural analyses (Bhardwaj et al. 2015b; Tran et al. 2015; Bui et al. 2016; Nguyen et al. 2018; Nguyen et al. 2019) and optimization problems (Ha et al. 2010; Taheri and Hassani 2014; Lieu and Lee 2019; Wang et al. 2019). Most of the previous studies have their focus on deterministic structural systems. For stochastic problems, limited studies on SIGA are available in the literature, for example, Bhardwaj et al. (2015a) used Monte Carlo simulation fatigue crack growth of FGMs using extended isogeometric analysis. Li et al. (2018a) proposed the spectral SIGA for linear elasticity problems. Nguyen et al. (2017) generated random fields by the spectral representation method to investigate the response variability of the buckling load of composite structures. Shahrokhabadi and Vahedifard (2018) combined isogeometric and generation random fields of water in the soil to model seepage in unsaturated soils. Zhang and Shibutani (2019) used polynomial chaos expansions to construct SIGA for uncertainty in shape. Ding et al. (2019) considered higher-order Taylor series of functions of random variables to propose the Isogeometric generalized nth order perturbation-based stochastic method. Eckert et al. (2020) developed a polynomial chaos method for an arbitrary random field in conjugate the standard isogeometric analysis for computational stochastic mechanics.

Almost all previous studies on relation stochastic isogeometric analysis involved random fields, discretization random fields used the Karhunen-Loève expansion or polynomial chaos method. The governing equations used these approach is quite complicated, so it needs to propose other approaches simpler than the approach mentioned above.

In the previous study, Hien and Noh (2017) dealt with the response variability of eigenvalues for the vibration problem of functionally graded plates with uncertain material properties. In this work, we focus on developing the SIGA for the static bending problem of functionally graded plates with uncertain elastic modulus. Based on the integration point method and perturbation technique to formulate the governing equations of SIGA, a strategic solution for the stochastic problem of static bending plate is presented. To demonstrate the appropriateness of the proposed scheme, numerical example analyses are performed using the proposed SIGA, and the results are compared with those given in the previous studies and with the results given by Monte Carlo simulation as well.

\section{THE NURBS FUNCTIONS}

In this section, the B-spline basis functions and nonuniform rational B-spline (NURBS) functions are briefly reviewed (Piegl and Tiller 1997; Cottrell et al. 2009). A knot vector $\Xi$ is defined as a set of coordinates in the domain $\xi \in[0,1]$ with the polynomial order $p$ :

$\Xi=\left\{\xi_{1}, \xi_{2}, \cdots, \xi_{n+p+1}\right\}, \quad \xi_{i} \leq \xi_{i+1}, \xi_{i} \in R$

The B-spline basis functions are defined recursively starting with piecewise constants $(p=0,1,2, \ldots)$ as follows:

$$
\begin{aligned}
& N_{i, 0}(\xi)= \begin{cases}1 & \text { if } \xi_{i} \leqslant \xi<\xi_{i+1}, \\
0 & \text { otherwise. }\end{cases} \\
& N_{i, p}(\xi)=N_{i+1, p-1}(\xi) \frac{\xi_{i+p+1}-\xi}{\xi_{i+p+1}-\xi_{i+1}}+N_{i, p-1}(\xi) \frac{\xi-\xi_{i}}{\xi_{i+p}-\xi_{i}}
\end{aligned}
$$

The B-spline surfaces create by the tensor product of basis functions in two parametric dimensions of $\eta$ and $\xi$ with two knot vectors $\Phi=\left\{\eta_{1}, \eta_{2}, \ldots, \eta_{\mathrm{m}+\mathrm{q}+1}\right\}$ and $\Xi=\left\{\xi_{1}, \xi_{2}, \ldots, \xi_{\mathrm{n}+\mathrm{p}+1}\right\}$ as form:

$$
\mathbf{S}(\xi, \eta)=\sum_{i=1}^{n} \sum_{j=1}^{m} N_{i, p}(\xi) M_{j, q}(\eta) \mathbf{P}_{i, j}
$$

where the B-spline basis functions $N_{i, p}(\xi)$ and $M_{j, q}(\eta)$. 
The NURBS functions are constructed from the B-spline basis functions by adding individual weights $w_{I}$ at each control point, and are expressed as:

$$
R_{i, p}(\xi)=\frac{N_{i, p}(\xi) w_{i}}{\sum_{I=1}^{n} N_{I, p}^{b}(\xi) w_{I}}
$$

The non-uniform rational B-spline curve is constructed by combining the rational basis functions and coefficients at control points:

$C(\xi)=\sum_{i=1}^{n} R_{i, p}(\xi) \boldsymbol{B}_{i}$

In general, the NURBS surface of order $p$ in the $\xi$ direction and order $q$ in the $\eta$ direction can be expressed as:

$\mathbf{S}(\xi, \eta)=\sum_{I=1}^{m \times n} R_{I}(\xi, \eta) \boldsymbol{B}_{I} \quad$ with $R_{I}(\xi, \eta)=\frac{N_{I}^{b} w_{I}}{\sum_{I=1}^{m \times n} N_{I}^{b}(\xi, \eta) w_{I}}$

\section{ISOGEOMETRIC FORMULATIONS FOR FGM PLATES BASED ON REFINED PLATE THEORY}

Consider a rectangular plate made of functionally graded material as shown in Figure 1.

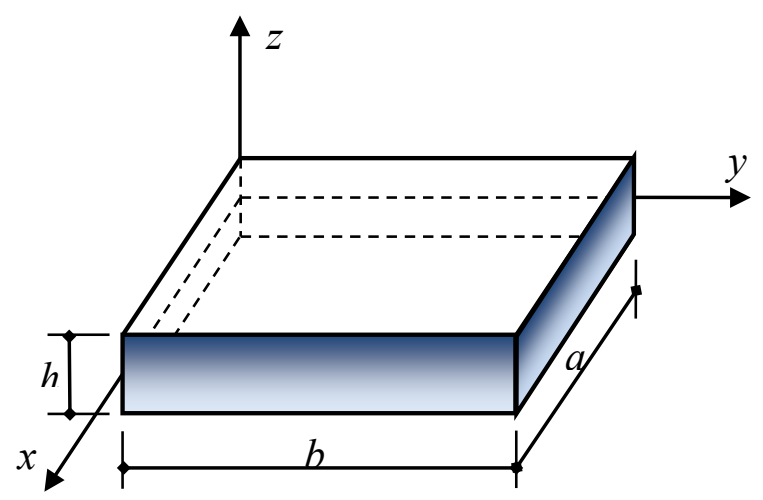

Figure 1 Geometry of functionally graded plate

In this work, the refined plate theory (RPT) (Shimpi and Patel 2006) which enables taking into account the shear deformation effect is used. Thus, the displacements field at $(x, y, z)$ in the FGM plates can be expressed as follows:

$$
\begin{aligned}
& U=u_{0}-z \frac{\partial w_{b}}{\partial x}+\left[\frac{1}{4} z-\frac{5}{3} z\left(\frac{z}{h}\right)^{2}\right] \frac{\partial w_{s}}{\partial x} \\
& V=v_{0}-z \frac{\partial w_{b}}{\partial y}+\left[\frac{1}{4} z-\frac{5}{3} z\left(\frac{z}{h}\right)^{2}\right] \frac{\partial w_{s}}{\partial y} \\
& W=w_{b}+w_{s}
\end{aligned}
$$

where $u_{0}, v_{0}, w_{b}, w_{s}$ are the displacement components on the mid-plane.

By differentiating Eq. (7), the linear strains can be obtained as: 
$\boldsymbol{\varepsilon}=\boldsymbol{\varepsilon}_{0}+z \boldsymbol{\kappa}_{b}+\Psi(z) \boldsymbol{\kappa}_{s}, \boldsymbol{\gamma}=\chi(z) \boldsymbol{\varepsilon}_{s}$

where:

$\boldsymbol{\varepsilon}=\left\{\begin{array}{l}\varepsilon_{x} \\ \varepsilon_{y} \\ \gamma_{x y}\end{array}\right\}, \boldsymbol{\varepsilon}_{0}=\left\{\begin{array}{c}\frac{\partial u_{0}}{\partial x} \\ \frac{\partial v_{0}}{\partial y} \\ \frac{\partial u_{0}}{\partial y}+\frac{\partial v_{0}}{\partial x}\end{array}\right\}, \boldsymbol{\kappa}_{b}=\left\{\begin{array}{c}-\frac{\partial^{2} w_{b}}{\partial x^{2}} \\ -\frac{\partial^{2} w_{b}}{\partial y^{2}} \\ -2 \frac{\partial^{2} w_{b}}{\partial x \partial y}\end{array}\right\}$,

$\boldsymbol{\kappa}_{s}=\left\{\begin{array}{l}-\frac{\partial^{2} w_{s}}{\partial x^{2}} \\ -\frac{\partial^{2} w_{s}}{\partial y^{2}} \\ -2 \frac{\partial^{2} w_{s}}{\partial x \partial y}\end{array}\right\}, \gamma=\left\{\begin{array}{l}\gamma_{y z} \\ \gamma_{x z}\end{array}\right\}, \boldsymbol{\varepsilon}_{s}=\left\{\begin{array}{c}\frac{\partial w_{s}}{\partial y} \\ \frac{\partial w_{s}}{\partial x}\end{array}\right\}$

and

$\Psi(z)=-\frac{1}{4} z+\frac{5}{3} z\left(\frac{z}{h}\right)^{2}, \chi(z)=5\left[\frac{1}{4}-\left(\frac{z}{h}\right)^{2}\right]$

We consider an FGM plate made from a mixture of ceramic and metal with the subscripts $m$ and $c$, respectively. The elastic modulus is assumed to be a random field in the plane of the plate:

$E=[1+r(x, y)] E_{0}(z)$

where $r(x, y)$ is the homogeneous random field with zero mean and $E_{0}(z)$ is assumed as:

$E_{0}(z)=\left(E_{c}-E_{m}\right)\left(\frac{z}{h}+\frac{1}{2}\right)^{p}+E_{m}$

where $p$ is the power index of the volume fraction.

The auto-correlation function of the random fields $r(x, y)$ :

$R_{i j}=R\left[r\left(x_{i}, y_{i}\right), r\left(x_{j}, y_{j}\right)\right]=\mathrm{E}\left[\left(r\left(x_{i}, y_{i}\right)-\mu_{r}\left(x_{i}, y_{i}\right)\right)\left(r\left(x_{j}, y_{j}\right)-\mu_{r}\left(x_{j}, y_{j}\right)\right)\right]$

The linear constitutive relations of FGM plates:

$\left\{\begin{array}{l}\sigma_{x} \\ \sigma_{y} \\ \sigma_{y z} \\ \sigma_{x z} \\ \sigma_{x y}\end{array}\right\}=[1+r(x, y)]\left[\begin{array}{ccccc}Q_{11} & Q_{12} & 0 & 0 & 0 \\ Q_{12} & Q_{22} & 0 & 0 & 0 \\ 0 & 0 & Q_{44} & 0 & 0 \\ 0 & 0 & 0 & Q_{55} & 0 \\ 0 & 0 & 0 & 0 & Q_{66}\end{array}\right]\left\{\begin{array}{l}\varepsilon_{x} \\ \varepsilon_{y} \\ \gamma_{y z} \\ \gamma_{x z} \\ \gamma_{x y}\end{array}\right\}$ 
where

$Q_{11}=\frac{E_{0}(z)}{1-v^{2}}, Q_{22}=Q_{11}, Q_{12}=\frac{v E_{0}(z)}{1-v^{2}}$

$Q_{44}=G_{23}=\frac{E_{0}(z)}{2(1+v)}, Q_{55}=G_{13}=\frac{E_{0}(z)}{2(1+v)}, Q_{66}=G_{12}=\frac{E_{0}(z)}{2(1+v)}$

The displacement field $\tilde{\boldsymbol{U}}$ of the plate is approximated by the NURBS functions and the vector of nodal $\boldsymbol{U}_{P}$ at the control point $P$ :

$\tilde{\boldsymbol{U}}(\xi, \eta)=\sum_{P=1}^{m \times n} R_{P}(\xi, \eta) \boldsymbol{U}_{P}$

where:

$\boldsymbol{U}_{P}=\left\{\begin{array}{c}u_{0 P} \\ v_{0 P} \\ w_{0 P}^{b} \\ w_{0 P}^{s}\end{array}\right\}$

The strains can be computed as:

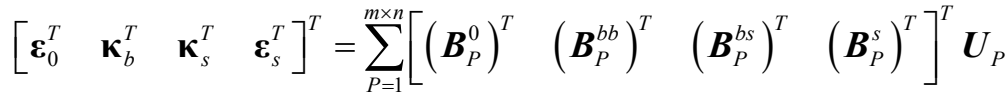

where

$\boldsymbol{B}_{P}^{0}=\left[\begin{array}{ccc}R_{P, x} & 0 & 0 \\ 0 & R_{P, y} & 0 \\ R_{P, y} & R_{P, x} & 0\end{array}\right], \boldsymbol{B}_{P}^{b b}=-\left[\begin{array}{ccc}0 & 0 & R_{P, x x} \\ 0 & 0 & R_{P, y y} \\ 0 & 0 & 2 R_{P, x y}\end{array}\right]$,

$\boldsymbol{B}_{P}^{b s}=-\left[\begin{array}{ccc}0 & 0 & R_{P, x x} \\ 0 & 0 & R_{P, y y} \\ 0 & 0 & 2 R_{P, x y}\end{array}\right], \boldsymbol{B}_{P}^{s}=\left[\begin{array}{ccc}0 & 0 & R_{P, x} \\ 0 & 0 & R_{P, y}\end{array}\right]$.

The weak form for static for bending of FGM plates can be written as to form:

$\int_{\Omega} \boldsymbol{\varepsilon}^{T}[1+r(x, y)] \boldsymbol{D}^{b} \delta \boldsymbol{\varepsilon} d \Omega+\int_{\Omega} \boldsymbol{\gamma}^{T}[1+r(x, y)] \boldsymbol{A}^{s} \delta \boldsymbol{\gamma} d \Omega=\int_{\Omega} q \delta w d \Omega$

where the stiffness matrices $\boldsymbol{A}^{s}, \boldsymbol{D}^{b}$ are given as follows: 


$$
\begin{aligned}
\boldsymbol{D}^{b} & =\left[\begin{array}{ccccccccc}
A_{11} & A_{12} & 0 & B_{11} & B_{12} & 0 & B_{11}^{s} & B_{12}^{s} & 0 \\
A_{12} & A_{22} & 0 & B_{12} & B_{22} & 0 & B_{12}^{s} & B_{22}^{s} & 0 \\
0 & 0 & A_{66} & 0 & 0 & B_{66} & 0 & 0 & B_{66}^{s} \\
B_{11} & B_{12} & 0 & D_{11} & D_{12} & 0 & D_{11}^{s} & D_{12}^{s} & 0 \\
B_{12} & B_{22} & 0 & D_{12} & D_{22} & 0 & D_{12}^{s} & D_{22}^{s} & 0 \\
0 & 0 & B_{66} & 0 & 0 & D_{66} & 0 & 0 & D_{66}^{s} \\
B_{11}^{s} & B_{12}^{s} & 0 & D_{11}^{s} & D_{12}^{s} & 0 & H_{11}^{s} & H_{12}^{s} & 0 \\
B_{12}^{s} & B_{22}^{s} & 0 & D_{12}^{s} & D_{22}^{s} & 0 & H_{12}^{s} & H_{22}^{s} & 0 \\
0 & 0 & B_{66}^{s} & 0 & 0 & D_{66}^{s} & 0 & 0 & H_{66}^{s}
\end{array}\right] \\
\boldsymbol{A}^{s} & =\left[\begin{array}{cc}
A_{44}^{s} & 0 \\
0 & A_{55}^{s}
\end{array}\right]
\end{aligned}
$$

where $A_{i j}, B_{i j}$, etc., are the uncertain plate stiffness, defined by

$\left\{A_{i j}, B_{i j}, D_{i j}\right\}=\int_{-h / 2}^{h / 2}\left\{1, z, z^{2}\right\} Q_{i j} d z, \quad(i=1,2,6)$

$B_{i j}^{s}=\int_{-h / 2}^{h / 2} \psi Q_{i j} d z, \quad(i=1,2,6)$

$D_{i j}^{s}=\int_{-h / 2}^{h / 2} z \psi Q_{i j} d z, \quad(i=1,2,6)$

$H_{i j}^{s}=\int_{-h / 2}^{h / 2} \psi^{2} Q_{i j} d z, \quad(i=1,2,6)$

$A_{i j}^{s}=\int_{-h / 2}^{h / 2} \chi Q_{i j} d z, \quad(i=4,5)$

Substituting Eq. (18) into Eq. (20), we obtain the governing equation for bending of FGM plates in the following form:

$K \boldsymbol{U}=\boldsymbol{F}(23)$

where $\boldsymbol{K}, \boldsymbol{U}$, and $\boldsymbol{F}$ denote stiffness matrix, generalized displacement, and force vector, respectively.

The global stiffness matrix $\boldsymbol{K}$ including the random field of elastic modulus is given by:

$\boldsymbol{K}=\int_{\Omega}\left\{\left\{\begin{array}{lll}\boldsymbol{B}^{0} & \boldsymbol{B}^{b b} & \boldsymbol{B}^{b s}\end{array}\right\} \boldsymbol{D}^{b}\left\{\begin{array}{c}\boldsymbol{B}^{0} \\ \boldsymbol{B}^{b b} \\ \boldsymbol{B}^{b s}\end{array}\right\}+\left(\boldsymbol{B}^{s}\right)^{T} \boldsymbol{A}^{s} \boldsymbol{B}^{s}\right\} \mathrm{d} \Omega$

and the load vector is given by:

$\boldsymbol{F}=\int_{\Omega} q \boldsymbol{R} \mathrm{d} \Omega$

where

$\boldsymbol{R}=\left[\begin{array}{llll}0 & 0 & R_{P} & R_{P}\end{array}\right]$ 


\section{STOCHASTIC ISOGEOMETRIC ANALYSIS FOR STATIC BENDING OF PLATES}

Similar to numerical methods applied in the stochastic analyses such as SFEM and stochastic meshless method, the SIGA also necessitates the discretization of random fields into a vector of random variables. Various methods for the discretization of random fields have been developed in the literature, for example, the nodal point method (Liu, Belytschko et al. 1986; Michael Kleiber and Hien 1992), the integration point method (Brenner and Bucher 1995; Matthies et al. 1997), the local averaging method (Vanmarcke and Grigoriu 1983), the Karhunen-Loève expansion (Ghanem and Spanos 1991; Ngah and Young 2007; Chen and Guedes Soares 2008; Druesne et al. 2016; Li et al. 2018b) and the weighted integral approach (Takada 1990; Deodatis 1991; Noh 2005). In this work, the random field of elastic modulus is discretized following the integration point method, i.e., the random field is approximated at Gauss points of each element.

Computation of the stiffness matrix in Eq.(24) using Gaussian integration quadrature as form:

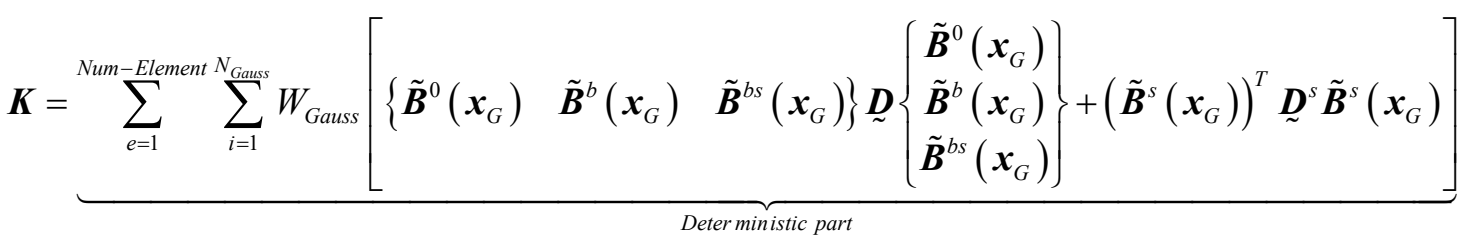

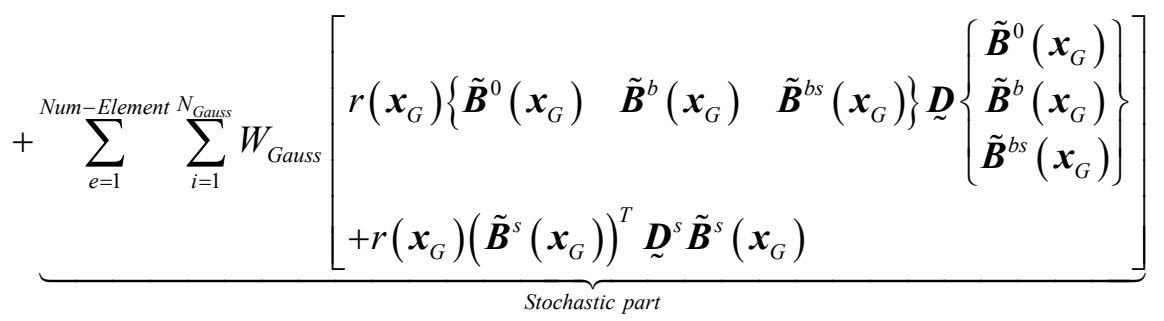

where $W_{\text {Gauss }}$ is weights of the Gaussian quadrature. In Eq.(27), the number of Gauss points equals the number of random variables. The set of $N$ random variables by discretization random field can be written in the form:

$\boldsymbol{r}=\left\{r_{1}\left(x_{1}, y_{1}\right), r_{2}\left(x_{2}, y_{2}\right), \ldots r_{N}\left(x_{N}, y_{N}\right)\right\}$

The equilibrium equation of the SIGA for static bending of FGM plates is:

$\boldsymbol{K}\left(r_{1}, r_{2}, \ldots r_{N}\right) \boldsymbol{U}\left(r_{1}, r_{2}, \ldots r_{N}\right)=\boldsymbol{F}$

We assume that all components of the random field $\boldsymbol{r}$ are small. Taking the Taylor series expansion at $\boldsymbol{r}=\mathbf{0}$, i.e., at the mean of $r$, gives:

$$
\begin{aligned}
& \boldsymbol{K}\left(r_{1}, r_{2}, \ldots r_{N}\right)=\boldsymbol{K}_{0}+\sum_{i=1}^{N} \boldsymbol{K}_{, i} r_{i}+\frac{1}{2} \sum_{i, j=1}^{N} \boldsymbol{K}_{, i j} r_{i} r_{j}+\ldots \\
& \boldsymbol{U}\left(r_{1}, r_{2}, \ldots r_{N}\right)=\boldsymbol{U}_{0}+\sum_{i=1}^{N} \boldsymbol{U}_{, i} r_{i}+\frac{1}{2} \sum_{i, j=1}^{N} \boldsymbol{U}_{, i j} r_{i} r_{j}+\ldots
\end{aligned}
$$

In which:

$$
\begin{gathered}
\boldsymbol{K}_{0}=\left.\boldsymbol{K}\right|_{r_{1}=0, r_{2}=0, \ldots, r_{N}=0} ; \quad \boldsymbol{K}_{, i}=\left.\frac{\partial \boldsymbol{K}}{\partial r_{i}}\right|_{r_{1}=0, r_{2}=0, \ldots, r_{N}=0} ; \quad \boldsymbol{K}_{, i j}=\left.\frac{\partial^{2} \boldsymbol{K}}{\partial r_{i} \partial r_{j}}\right|_{r_{1}=0, r_{2}=0, \ldots, r_{N}=0} \\
\boldsymbol{U}_{0}=\left.\boldsymbol{U}\right|_{r_{1}=0, r_{2}=0, \ldots r_{N}=0} ; \quad \boldsymbol{U}_{, i}=\left.\frac{\partial \boldsymbol{U}}{\partial r_{i}}\right|_{r_{1}=0, r_{2}=0, \ldots, r_{N}=0} ; \quad \boldsymbol{U}_{, i j}=\left.\frac{\partial^{2} \boldsymbol{U}}{\partial r_{i} \partial r_{j}}\right|_{r_{1}=0, r_{2}=0, \ldots, r_{N}=0}
\end{gathered}
$$


Substituting Eq. (30) into Eq. (29) and then comparing terms of the same order obtains the perturbation equation: For the zeroth-order perturbation equation:

$$
\boldsymbol{K}_{0} \boldsymbol{U}_{0}=\boldsymbol{F}_{0}
$$

For the first-order perturbation equation:

$$
\boldsymbol{K}_{0} \boldsymbol{U}_{, i}=-\boldsymbol{K}_{, i} \boldsymbol{U}_{0}
$$

For the second-order perturbation equation:

$\boldsymbol{K}_{0} \boldsymbol{U}_{, i j}=-\boldsymbol{K}_{, i j} \boldsymbol{U}_{0}-\boldsymbol{K}_{, i} \boldsymbol{U}_{, j}-\boldsymbol{K}_{, j} \boldsymbol{U}_{, i}$

Let $\mu_{U}=\mathrm{E}(\boldsymbol{U})$ and $\operatorname{Cov}_{U}=\mathrm{E}\left[\left(\boldsymbol{U}-\mu_{U}\right)\left(\boldsymbol{U}-\mu_{U}\right)^{T}\right]$ sign the mean and covariance of the displacement $\boldsymbol{U}$, respectively.

Using the expectation operator on Eq. (30), the solutions of the first-order perturbation technique for the displacement are:

$$
\begin{aligned}
& \mu_{U}^{(I)}=\mathrm{E}\left(\boldsymbol{U}_{0}+\sum_{i=1}^{N} \boldsymbol{U}_{, i} r_{i}\right)=\boldsymbol{U}_{0} \\
& \operatorname{Cov}_{U}^{(l)}=\mathrm{E}\left[\left(\boldsymbol{U}-\mu_{U}\right)\left(\boldsymbol{U}-\mu_{U}\right)^{T}\right]=\mathrm{E}\left[\left(\boldsymbol{U}_{0}+\sum_{i=1}^{N} \boldsymbol{U}_{, i} r_{i}-\boldsymbol{U}_{0}\right)\left(\boldsymbol{U}_{0}+\sum_{j=1}^{N} \boldsymbol{U}_{, j} r_{j}-\boldsymbol{U}_{0}\right)^{T}\right] \\
& =\mathrm{E}\left[\left(\sum_{i=1}^{N} \boldsymbol{U}_{, i} r_{i}\right)\left(\sum_{j=1}^{N} \boldsymbol{U}_{, j} r_{j}\right)\right]=\sum_{i=1}^{N} \sum_{j=1}^{N} \boldsymbol{U}_{, i} \boldsymbol{U}_{, j} R_{i j}
\end{aligned}
$$

where $\boldsymbol{R}_{i j}$ is the covariance matrix of a random variable $\boldsymbol{r}$ and $N$ is the number of random variables.

The second-order perturbation solutions are:

$\mu_{U}^{(2)}=\mathrm{E}\left(\boldsymbol{U}_{0}+\sum_{i=1}^{N} \boldsymbol{U}_{, i} r_{i}+\frac{1}{2} \sum_{i, j=1}^{N} \boldsymbol{U}_{, i j} r_{i} r_{j}\right)=\boldsymbol{U}_{0}+\frac{1}{2} \sum_{i=1}^{N} \sum_{j=1}^{N} \boldsymbol{U}_{, i j} R_{i j}$

$\operatorname{Cov}_{U}^{(2)}=\mathrm{E}\left[\left(\boldsymbol{U}-\mu_{U}^{(2)}\right)\left(\boldsymbol{U}-\mu_{U}^{(2)}\right)^{T}\right]$

$=\mathrm{E}\left[\begin{array}{l}\left(\boldsymbol{U}_{0}+\sum_{i=1}^{N} \boldsymbol{U}_{, i} r_{i}+\frac{1}{2} \sum_{i, j=1}^{N} \boldsymbol{U}_{, i j} r_{i} r_{j}-\boldsymbol{U}_{0}-\frac{1}{2} \sum_{i=1}^{N} \sum_{j=1}^{N} \boldsymbol{U}_{, i j} R_{i j}\right) \times \\ \left(\boldsymbol{U}_{0}+\sum_{i=1}^{N} \boldsymbol{U}_{, i} r_{i}+\frac{1}{2} \sum_{i, j=1}^{N} \boldsymbol{U}_{, i j} r_{i} r_{j}-\boldsymbol{U}_{0}-\frac{1}{2} \sum_{i=1}^{N} \sum_{j=1}^{N} \boldsymbol{U}_{, i j} R_{i j}\right)^{T}\end{array}\right]$

$=\sum_{i=1}^{N} \sum_{j=1}^{N} \boldsymbol{U}_{, i} \boldsymbol{U}_{, j} R_{i j}+\frac{1}{4} \sum_{i=1}^{N} \sum_{j=1}^{N} \sum_{k=1}^{N} \sum_{l=1}^{N} \boldsymbol{U}_{, i j} \boldsymbol{U}_{, k l}\left(R_{i k} R_{j l}+R_{i l} R_{j k}\right)$ 
The generalized displacement vector, $\boldsymbol{U}$, represents nodal parameters at control points; it is not the actual displacements at nodes. Using the relation between $\boldsymbol{U}$ and $\tilde{\boldsymbol{U}}$ in Eq. (16), the mean vector and covariance matrix can be obtained as:

$$
\begin{aligned}
& \mu_{\tilde{U}}=\Theta \mu_{U} \\
& \operatorname{Cov}_{\tilde{U}}=\boldsymbol{\Theta} \operatorname{Cov}_{U} \Theta^{T}
\end{aligned}
$$

where $\Theta$ is a transformation matrix which is calculated via the relation between $\boldsymbol{U}$ and $\tilde{\boldsymbol{U}}$ in Eq.(16).

The response variability can be represented by means of the coefficient of variation (COV), which is defined as a ratio of the standard deviation to the mean of displacement as follows:

$$
C O V=\frac{\sqrt{\operatorname{Var}_{\tilde{U}}}}{\left|\mu_{\tilde{U}}\right|}
$$

The numerical solution procedure for a nonstatistical approach is shown on the flow chart in Figure 2. The main steps of the process solving can briefly be described as follows:

1) The first step is to input data and discretize the structural domain.

2) The second step is to calculate the deterministic part of the stiffness matrix and load vector.

3) The third step is to solve the zeroth perturbation equation in Eq. (32) to find out displacements, strains, stresses.

4) The fourth step is to find the first-order perturbation solution for determining the mean and covariance matrix of the displacement parameter.

5) The fifth step is to find the second-order perturbation solution for determining the mean and covariance matrix of the displacement parameter.

6) The final step is to find the transformation matrix and then employ a loop to calculate the mean and covariance matrix of real displacements in Eq. (38).

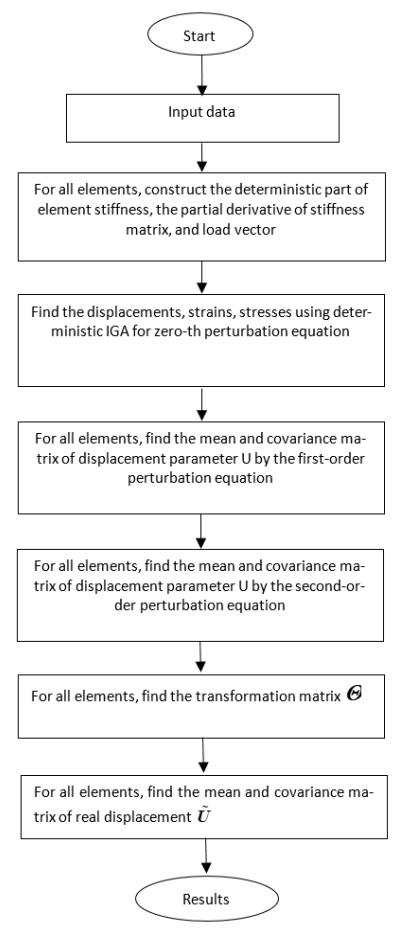

Figure 2 Schematic flow chart for nonstatistical approach by SIGA 


\section{MONTE CARLO SIMULATION}

In verifying the proposed SIGA, the crude Monte Carlo simulation is adopted and performed. The spectral representation method reviewed by Shinozuka and Deodatis (Shinozuka and Deodatis 1991; Shinozuka and Deodatis 1996) is well suited for the numerical generation of random sample functions of the Gaussian zero-mean homogeneous random fields.

The Gaussian zero-mean homogeneous random fields $r(x, y)$ can be represented by the summation expression for the random sample Shinozuka and Deodatis (1996):

$$
r(x, y)=\sqrt{2} \sum_{n_{1}=0}^{N_{1}} \sum_{n_{2}=0}^{N_{2}}\left[A_{n_{1} n_{2}}^{(1)} \cos \left(\omega_{1_{n 1}} x+\omega_{2_{n 2}} y+\varphi_{n_{1} n_{2}}^{(1)}\right)+A_{n_{1} n_{2}}^{(1)} \cos \left(\omega_{1_{n 1}} x-\omega_{2_{n 2}} y+\varphi_{n_{1} n_{2}}^{(2)}\right)\right]
$$

where:

$$
\begin{aligned}
& A_{n_{1} n_{2}}^{(1)}=\sqrt{2 S_{f f}\left(\omega_{1 n_{1}}, \omega_{2 n_{2}}\right) \Delta \omega_{1} \Delta \omega_{2}} \\
& A_{n_{1} n_{2}}^{(2)}=\sqrt{2 S_{f f}\left(\omega_{1 n_{1}},-\omega_{2 n_{2}}\right) \Delta \omega_{1} \Delta \omega_{2}} \\
& \omega_{1 n_{1}}=n_{1} \Delta \omega_{1} ; \quad \omega_{2 n_{2}}=n_{2} \Delta \omega_{2} \\
& \Delta \omega_{1}=\frac{\omega_{1 u}}{N_{1}} ; \Delta \omega_{2}=\frac{\omega_{2 u}}{N_{2}}
\end{aligned}
$$

The phase angles $\varphi_{n_{1} n_{2}}^{(1)}, \varphi_{n_{1} n_{2}}^{(2)}$ are uniformly distributed in the range of $[0,2 \pi]$. The upper cutoff limit of wavenumbers $\omega_{1 u}, \omega_{2 u}$ in the spectral density function $S_{f f}\left(\omega_{1}, \omega_{2}\right)$ can be determined by

$$
\int_{0}^{\omega_{1 u}} \int_{-\omega_{2 u}}^{\omega_{2 u}} S_{f f}\left(\omega_{1}, \omega_{2}\right) d \omega_{1} d \omega_{2}=(1-\varepsilon) \int_{0}^{\infty} \int_{-\infty}^{\infty} S_{f f}\left(\omega_{1}, \omega_{2}\right) d \omega_{1} d \omega_{2}
$$

Here, $\varepsilon$ is set to be 0.001 in the numerical generation.

With each random sample, the stiffness matrix is determined to contain the elastic modulus which is expressed as a cosine series as given in Eq.(40). The computation of the stiffness matrix is more complex due to the variable elastic modulus. It can approximately be calculated by the Gaussian quadrature for the integration of the stiffness matrix. Repeated solutions are needed for each sample to obtain the statistical properties of responses of the corresponding structure.

\section{NUMERICAL EXAMPLE}

\subsection{Numerical tests}

The accuracy of the proposed analysis scheme can be verified if the method predicts well the previous reports given in the literature, in particular for the structures composed of isotropic materials. Even though the formulation is given for the FGM plates, it can also be applied to the analysis of the isotropic plates if we use corresponding parameters.

The first example is a square plate subject to a uniformly distributed load having a magnitude of 1.0 (Figure 3). The data of this example follows the first example in the previous study by Choi and Noh (1996). The boundary conditions considered are two cases: simple support and clamped support. Because of the symmetry in the shape and the applied load of the structure, a quarter model is adopted (Figure 3). 


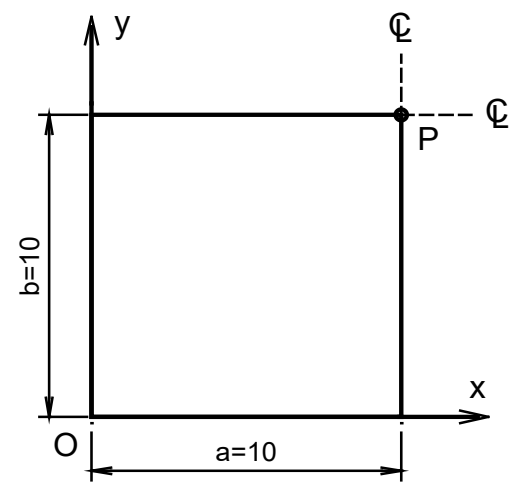

Figure $3 \mathrm{~A}$ quarter model of the plate

The mean Young's modulus is chosen to have the same value as used in the literature Choi and Noh (1996): $E_{0}=10.29 \times 10^{3}$. Poisson's ratio is 0.30 . The thickness of the plate is assumed as 1.0 . The auto-correlation function is assumed as:

$R\left(\xi_{x}, \xi_{y}\right)=\sigma^{2} e^{-\frac{\left|\xi_{x}\right|}{d_{1}}-\frac{\left|\xi_{y}\right|}{d_{2}}}$

where, $\xi_{x}, \xi_{y}$ are components of the separation vector $\xi$ between two points in the domain of the structure. The corresponding correlation distances are denoted by $d_{1}, d_{2}$. The coefficient of variation of the random field $\sigma$ is assumed as 0.1 .

We provide the stochastic response at the center point of the structure (point $P$ in Figure 3 ). In the numerical analyses, we use 64 quadratic NURBS elements ( $8 \times 8$ mesh).

In Table 1, we compare the mean and standard deviation of center displacements of the plate obtained by three analyses: present approach - the first-order solution of the SIGA scheme, Monte Carlo simulation with 10,000 samples based on the IGA scheme, weighted integral method, and Monte Carlo simulation Choi and Noh (1996). It is observed that all the results of stochastic isogeometric analyses, irrespective of boundary conditions, show larger values than those obtained by the weighted integral method. It is noted that the MCS results are even larger for SIGA than for FEM, which shows the superiority of the IGA scheme over conventional finite element analysis.

Table 1 Comparison of SIGA results with Noh Choi and Noh (1996) in the case $d_{1}=d_{2}=2.0$

\begin{tabular}{|c|c|c|c|c|c|c|}
\hline \multirow[b]{2}{*}{ No. } & \multirow[b]{2}{*}{ Type analysis } & \multirow{2}{*}{$\begin{array}{l}\text { Modeling } \\
\text { (Meshes) }\end{array}$} & \multicolumn{2}{|c|}{ Simple support } & \multicolumn{2}{|c|}{ Clamped support } \\
\hline & & & Mean & $\begin{array}{l}\text { Standard } \\
\text { deviation }\end{array}$ & Mean & $\begin{array}{l}\text { Standard } \\
\text { deviation }\end{array}$ \\
\hline 1 & SIGA & $8 \times 8$ & 0.6966 & 0.0246 & 0.2190 & 0.0072 \\
\hline 2 & Weighted Integral & $6 \times 6$ & 0.6591 & 0.0225 & 0.2129 & 0.0068 \\
\hline 3 & Exact Sol (Deterministic) & - & 0.7277 & - & 0.2267 & - \\
\hline 4 & MCS (FEM-Noh) & $4 \times 4$ & 0.6621 & 0.0288 & 0.2133 & 0.0091 \\
\hline 5 & MCS (FEM-Noh) & $6 \times 6$ & 0.6621 & 0.0260 & 0.2134 & 0.0081 \\
\hline 6 & MSC (Present-IGA) & $8 \times 8$ & 0.7004 & 0.0296 & 0.2198 & 0.0088 \\
\hline
\end{tabular}

\subsection{The second-order analysis}

A simply supported square FGM plate, made of aluminum and alumina $\left(\mathrm{Al} / \mathrm{Al}_{2} \mathrm{O}_{3}\right)$, is considered. The means of material properties are chosen to be the same as (Hasani Baferani et al. 2011): $E_{m}=70 \mathrm{GPa}, \rho_{m}=2707 \mathrm{~kg} / \mathrm{m}^{3}$, $E_{c}=380 \mathrm{GPa}, \rho_{c}=3800 \mathrm{~kg} / \mathrm{m}^{3}$, Poisson's ratio of the plate is assumed to be constant through the thickness and is equal

to 0.3 . The plate is subjected to unit distributed load. The dimensions of the FGM plate are 
$a=0.2(\mathrm{~m}) ; b=0.2(\mathrm{~m}) ; h=\frac{a}{20}$. In modeling the structure, we adopt $8 \times 8$ cubic NURBS elements that are used in stiffness evaluation. The auto-correlation function of the random fields in elastic modulus is assumed as follows:

$R\left(\xi_{x}, \xi_{y}\right)=\sigma^{2} e^{-\frac{\left|\xi_{x}\right|}{d_{1}}-\frac{\left|\xi_{y}\right|}{d_{2}}}$

We consider a normalized displacement at the center of the plate:

$\bar{w}_{P}=D_{1} w_{P}$

where the stiffness parameter:

$D_{1}=\frac{10^{3} E_{c} h^{3}}{12\left(1-v^{2}\right) q_{0} a^{4}}$.

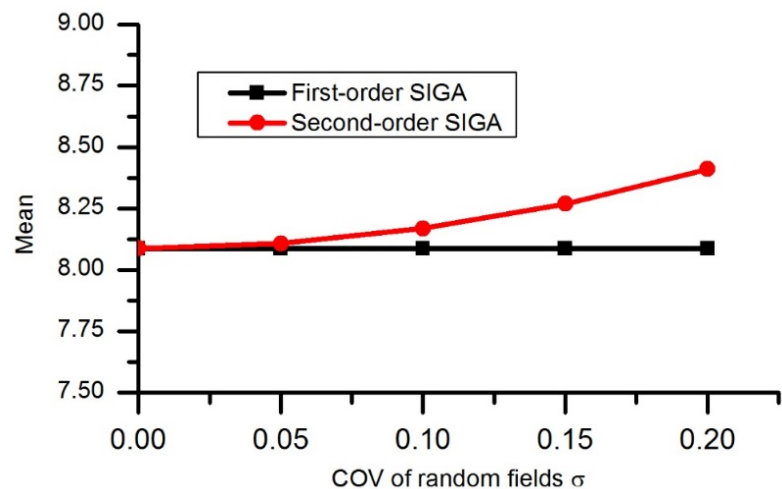

Figure 4: The first- and second-order SIGA solution for the mean of normalized displacement $w_{P}$

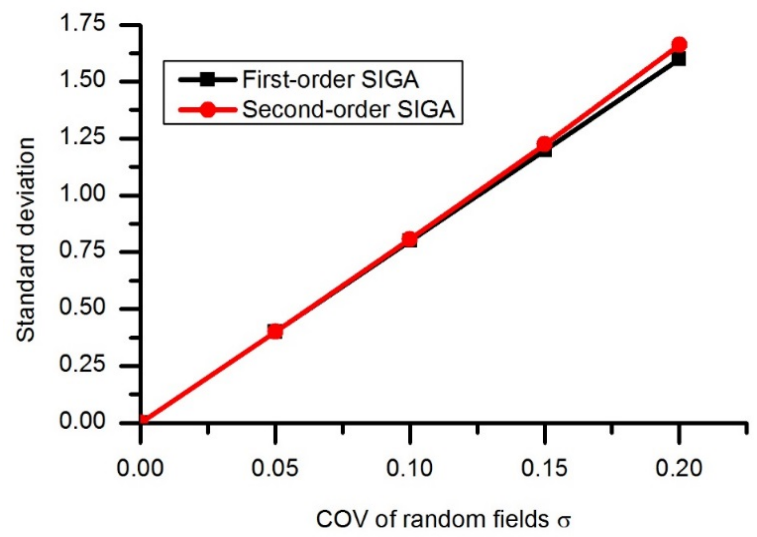

Figure 5: The first- and second-order SIGA solution for the standard deviation of normalized displacement $\mathcal{W}_{P}$

The mean and standard deviation of normalized displacement at the center of the FGM plate obtained by using the first- and second-order SIGA in terms of varying COV of the random field show in Figs. 4 and 5 . Here, the power index $p=1$ and the correlation distance $d_{1}=d_{2}=100 a$. It is clear from Figs. 4 and 5 that the effect of second-order stochastic isogeometric analyses becomes prominent as the coefficient of variation of the random field increases if we see from the viewpoint of the mean and the standard deviation, respectively. 


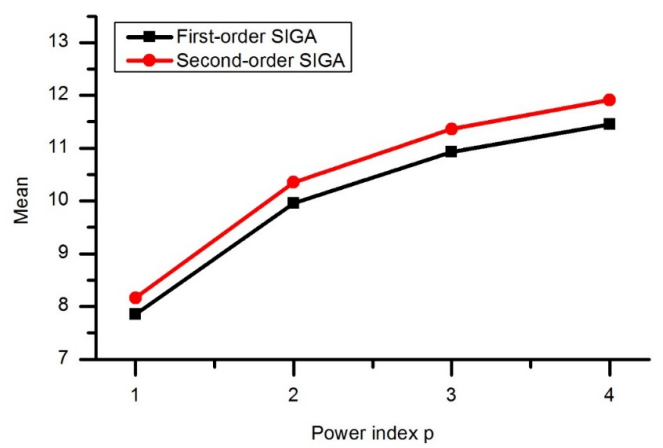

Figure 6: The first- and second-order SIGA solution for the mean of normalized displacement $w_{P}$ in terms of power index $p$

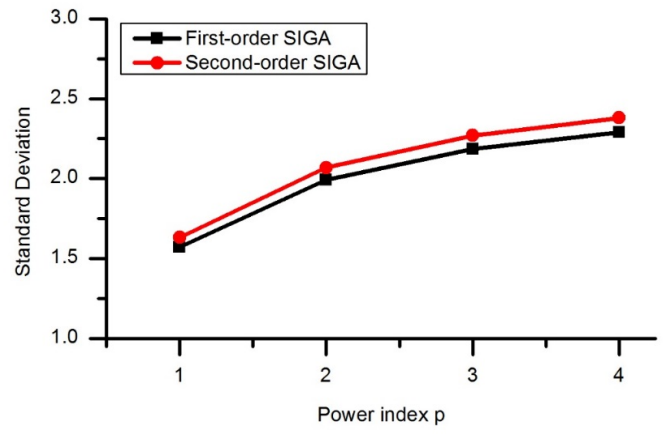

Figure 7: The first- and second-order SIGA solution for the standard deviation of normalized displacement $w_{P}$ in terms of power index $p$

Figures 6 and 7 show the first- and second-order SIGA solutions for the mean and the standard deviation of normalized displacement at the center of the FGM plate in terms of varying power index $p$ in Eq.(12). Here, we assume that the coefficient of variation of random fields $\sigma=0.2$, and the correlation distance $d_{1}=d_{2}=20 a$. It is clear that all the responses of the second-order SIGA are larger than those of the first-order SIGA. It is also observed that the mean and the standard deviation of normalized displacement increases, with a decreasing rate, as the power index $p$ increases.

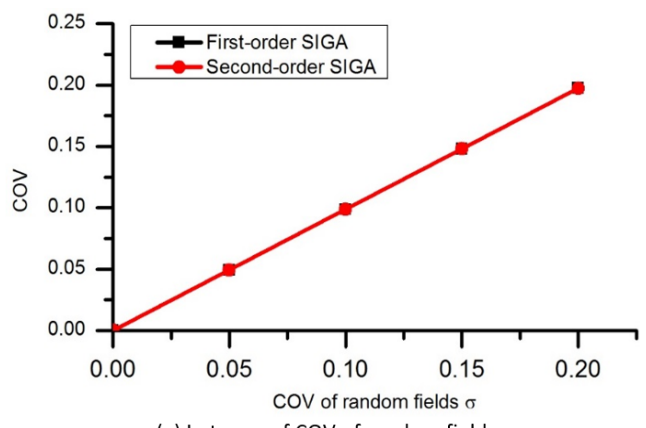

(a) In terms of COV of random field

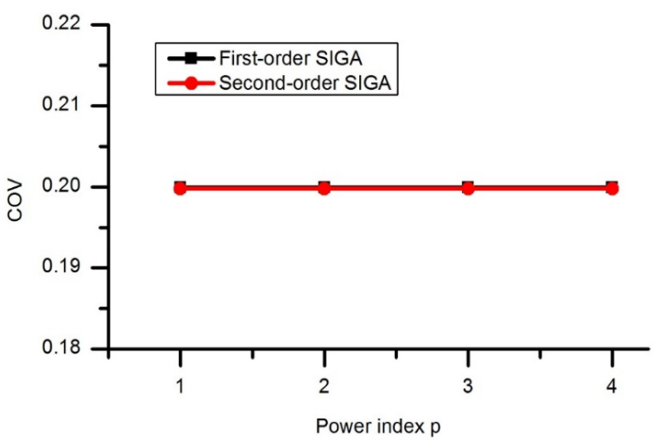

(b) In terms of power index $p$

Figure 8: The effect of COV of random field and power index $p$ on the COV of displacements 
However, we can note that the response COV is not affected by the order of the scheme adopted (first or second order), as shown in Figure 8, because the denominator (mean) and the numerator (standard deviation) of the response COV increase simultaneously with almost the same degree. It is clear that the effect of the power index $p$ on response COV in Fig. $8 \mathrm{~b}$ is very small. A similar trend is observed also for the clamped plate. The power index $p$ affects the mean and the standard deviation of the normalized displacement as shown in Figs. 6 and 7, however, it does not affect the responses COV.

\subsection{The effect of correlation distance ratio on the static bending of plates}

The effect of correlation distance ratio between two directions, $d_{2} / d_{1}$, on the response variability of displacements is considered in this part. We provide results obtained by using the first-order SIGA for the same plate used in Section 5.2.

The dimensions of FGM plates: $a=0.2(\mathrm{~m}), b=0.3(\mathrm{~m}), h=0.01(\mathrm{~m})$. The power index $p$ is fixed as 1.0. The effect of the correlation distance ratio $d_{2} / d_{1}$ on the response COV of the normalized displacement at the center of FGM plates with simple and fixed boundary conditions is represented in Figure 9 with the coefficient $\sigma=0.1$. It is obvious that the response COV goes up as the ratio $d_{2} / d_{1}$ increases.

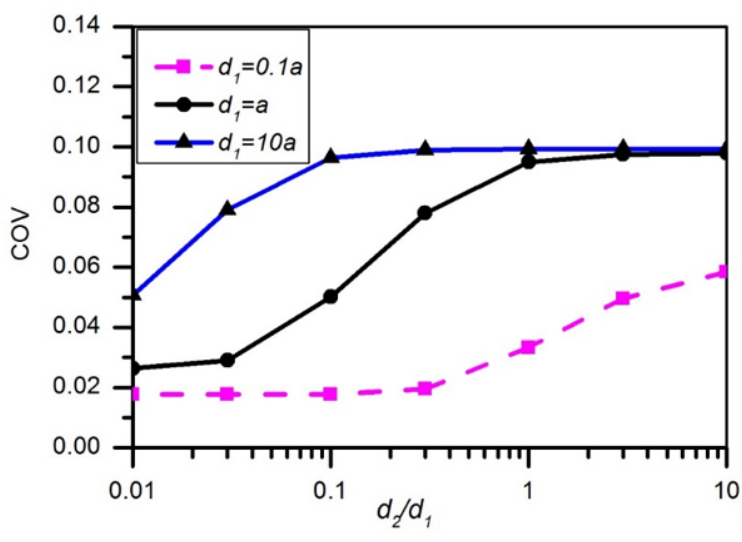

a) Simple plate

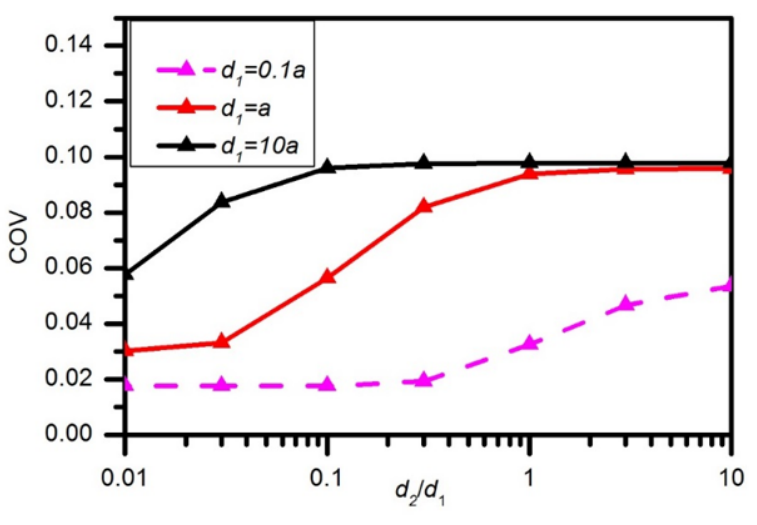

b) Fixed plate

Figure 9: Response COV \& correlation distance ratio $d_{2} / d_{1}$

\section{CONCLUSION}

SIGA is proposed for the static bending analysis of FGM plates with randomness in elastic modulus. In constructing the governing equations for the FGM plate, the effect of higher-order shear deformation is taken into account. Two dimensional random fields of elastic modulus are discretized using the integration point method to formulate the firstand second-order perturbation expansion of displacements and stiffness matrix. This approach is simpler than the Karhunen-Loève expansion approach or polynomial chaos approach in the Spectral stochastic isogeometric analysis. 
The first- and second-order perturbation analysis schemes are presented in conjunction with the standard IGA method. Also, Monte Calo simulation is presented by combining the numerical generation of random field using the spectral representation method and standard isogeometric analysis. The validation of the proposed stochastic analysis schemes is determined by comparing the mean, variance, and response variability of the deflection at the center of plates predicted by the proposed method with those from MCS and previous studies. The numerical examples clearly demonstrate that the proposed scheme shows good agreement with those from MCS and the previous research results in the literature, and furthermore give more accurate results. The effect of second-order analysis shown is significant when the degree of uncertainty is high in the random field under consideration. In particular, not only the deterministic analysis results but also the stochastic responses are observed to be improved due to the adoption of the IGA.

From the analyses considering various parameters of the given FGM plate problems, we observe the following: The effects of power index on the response COV of displacement is negligible. In contrast, the influence of power index $p$ on the standard deviation of displacement is prominent depending strongly on the value of $p$. The effect of correlation distance ratio on the response COV is obvious, and the response COV grows when the correlation distance ratio raises.

In this work, the SIGA which calculates the stochastic response considering the random field of material properties provides the advanced stochastic analysis of plates. The results of the stochastic analysis of structures can use for advanced design.

\section{Acknowledgment}

This research is funded by Vietnam National Foundation for Science and Technology Development (NAFOSTED) under grant number 107.01-2017.314

Author's Contributions: Writing - original draft, TD Hien; Writing - review \& editing, PC Nguyen.

Editor: Rogério José Marczak.

\section{References}

Arun, C.O., Rao, B.N. and Srinivasan, S.M. (2010), Stochastic meshfree method for elasto-plastic damage analysis, Computer Methods in Applied Mechanics and Engineering, 199(37-40), 2590-2606.

Bhardwaj, G., Singh, I.V. and Mishra, B.K. (2015a), Stochastic fatigue crack growth simulation of interfacial crack in bi-layered FGMs using XIGA, Computer Methods in Applied Mechanics and Engineering, 284, 186-229.

Bhardwaj, G., Singh, I.V., Mishra, B.K. and Bui, T.Q. (2015b), Numerical simulation of functionally graded cracked plates using NURBS based XIGA under different loads and boundary conditions, Composite Structures, 126, 347-359.

Brenner, C.E. and Bucher, C. (1995), A contribution to the SFE-based reliability assessment of nonlinear structures under dynamic loading, Probabilistic Engineering Mechanics, 10(4), 265-273.

Bui, T.Q., Hirose, S., Zhang, C., Rabczuk, T., Wu, C.-T., Saitoh, T. and Lei, J. (2016), Extended isogeometric analysis for dynamic fracture in multiphase piezoelectric/piezomagnetic composites, Mechanics of Materials, 97, 135-163.

Cavdar, O., Bayraktar, A., Cavdar, A. and Adanur, S. (2008), Perturbation based stochastic finite element analysis of the structural systems with composite sections under earthquake forces, Steel Compos. Struct. Steel and Composite Structures, 8(2), 129-144.

Chakraborty, A. and Rahman, S. (2008), Stochastic multiscale models for fracture analysis of functionally graded materials, Engineering Fracture Mechanics, 75(8), 2062-2086.

Chen, N.-Z. and Guedes Soares, C. (2008), Spectral stochastic finite element analysis for laminated composite plates, Computer Methods in Applied Mechanics and Engineering, 197(51-52), 4830-4839.

Choi, C. and Noh, H. (1996), Stochastic finite element analysis of plate structures by weighted integral method, Structural Engineering and Mechanics, 4(6), 703-715.

Cottrell, J.A., Hughes, T.J.R., Bazilevs, Y. and Wiley, I. (2009), Isogeometric analysis: toward integration of CAD and FEA, Wiley, Chichester, West Sussex, U.K.; Hoboken, NJ. 
Deodatis, G. (1991), Weighted Integral Method. I: Stochastic Stiffness Matrix, Journal of Engineering Mechanics, 117(8), 18511864.

Ding, C., Hu, X., Cui, X., Li, G., Cai, Y. and Tamma, K.K. (2019), Isogeometric generalized $\mathrm{n}$ th order perturbation-based stochastic method for exact geometric modeling of (composite) structures: Static and dynamic analysis with random material parameters, Computer Methods in Applied Mechanics and Engineering, 346, 1002-1024.

Druesne, F., Hamdaoui, M., Lardeur, P. and Daya, E.M. (2016), Variability of dynamic responses of frequency dependent viscoelastic sandwich beams with material and physical properties modeled by spatial random fields, Composite Structures, 152 , 316-323.

Eckert, C., Beer, M. and Spanos, P.D. (2020), A polynomial chaos method for arbitrary random inputs using B-splines, Probabilistic Engineering Mechanics, 60, 103051.

Galal, O.H., El-Tahan, W., El-Tawil, M.A. and Mahmoud, A.A. (2008), Spectral SFEM analysis of structures with stochastic parameters under stochastic excitation, Structural Engineering and Mechanics, 28(3), 281-294.

Ghanem, R.G. and Spanos, P.D. (1991), Spectral stochastic finite-element formulation for reliability-analysis, J. Eng. Mech.ASCE, 117(10), 2351-2372.

Graham, L.L. and Deodatis, G. (2001), Response and eigenvalue analysis of stochastic finite element systems with multiple correlated material and geometric properties, Probabilistic Engineering Mechanics, 16(1), 11-29.

Ha, L.T. and Khue, N.T.K. (2019), Free vibration of functionally graded porous nano beams, Transport and Communications Science Journal, 70(2), 95-103.

Ha, S.-H., Choi, K.K. and Cho, S. (2010), Numerical method for shape optimization using T-spline based isogeometric method, Struct Multidisc Optim, 42(3), 417-428.

Hafed, Z.S. and Zenkour, A.M. (2019), Hygro-thermo-mechanical bending of FG piezoelectric plates using quasi-3D shear and normal deformations theory, lajss Latin American Journal of Solids and Structures, 16(7).

Hasani Baferani, A., Saidi, A.R. and Ehteshami, H. (2011), Accurate solution for free vibration analysis of functionally graded thick rectangular plates resting on elastic foundation, Composite Structures, 93(7), 1842-1853.

Hien, T.D. (2020), A static analysis of nonuniform column by stochastic finite element method using weighted integration approach Transport and Communications Science Journal, 70(4), 359-367.

Hien, T.D. and Noh, H.-C. (2017), Stochastic isogeometric analysis of free vibration of functionally graded plates considering material randomness, Computer Methods in Applied Mechanics and Engineering, 318, 845-863.

Hosseini, S.M. and Shahabian, F. (2014), Stochastic analysis of elastic wave and second sound propagation in media with Gaussian uncertainty in mechanical properties using a stochastic hybrid mesh-free method, Structural Engineering and Mechanics, 49(1), 41-64.

Hughes, T.J.R., Cottrell, J.A. and Bazilevs, Y. (2005), Isogeometric analysis: CAD, finite elements, NURBS, exact geometry and mesh refinement, Computer Methods in Applied Mechanics and Engineering, 194(39-41), 4135-4195.

Jun, L., Chaoxing, S., Xiangshao, K., Xiaobin, L. and Weiguo, W. (2014), Stochastic response of an axially loaded composite Timoshenko beam exhibiting bending-torsion coupling, Archive of Applied Mechanics, 84(1), 109-122.

Kaminski, M. (2001), Stochastic second-order perturbation approach to the stress-based finite element method, International Journal of Solids and Structures, 38(21), 3831-3852.

Kim, H.M. and Inoue, J. (2004), A spectral stochastic element free Galerkin method for the problems with random material parameter, International Journal for Numerical Methods in Engineering, 61(11), 1957-1975.

Koizumi, M. (1997), FGM activities in Japan, Composite: Part B, 28(1-2), 1-4.

Li, K., Gao, W., Wu, D., Song, C. and Chen, T. (2018a), Spectral stochastic isogeometric analysis of linear elasticity, Computer Methods in Applied Mechanics and Engineering, 332, 157-190.

Li, K., Wu, D. and Gao, W. (2018b), Spectral stochastic isogeometric analysis for static response of FGM plate with material uncertainty, Thin-Walled Structures, 132, 504-521. 
Lieu, Q.X. and Lee, J. (2019), An isogeometric multimesh design approach for size and shape optimization of multidirectional functionally graded plates, Computer Methods in Applied Mechanics and Engineering, 343, 407-437.

Liu, W.K., Belytschko, T. and Mani, A. (1986), Random field finite elements, International Journal for Numerical Methods in Engineering, 23(10), 1831-1845.

Matthies, H.G., Brenner, C.E., Bucher, C.G. and Guedes Soares, C. (1997), Uncertainties in probabilistic numerical analysis of structures and solids-Stochastic finite elements, Structural Safety, 19(3), 283-336.

Michael Kleiber and Hien, T.D. (1992), The stochastic finite element method: basic perturbation technique and computer implementation, Wiley

Miyamoto, Y., Kaysser, W.A., Rabin, B.H., Kawasaki, A. and Ford, R.G. (1999), Functionally Graded Materials: Design, Processing and Applications, Springer

Ngah, M.F. and Young, A. (2007), Application of the spectral stochastic finite element method for performance prediction of composite structures, Composite Structures, 78(3), 447-456.

Nguyen, H.X., Duy Hien, T., Lee, J. and Nguyen-Xuan, H. (2017), Stochastic buckling behaviour of laminated composite structures with uncertain material properties, Aerospace Science and Technology, 66, 274-283.

Nguyen, T.N., Thai, C.H., Luu, A.-T., Nguyen-Xuan, H. and Lee, J. (2019), NURBS-based postbuckling analysis of functionally graded carbon nanotube-reinforced composite shells, Computer Methods in Applied Mechanics and Engineering, 347, 9831003.

Nguyen, T.N., Thai, C.H., Nguyen-Xuan, H. and Lee, J. (2018), NURBS-based analyses of functionally graded carbon nanotubereinforced composite shells, Composite Structures, 203, 349-360.

Noh, H.-C. (2004), A formulation for stochastic finite element analysis of plate structures with uncertain Poisson's ratio, Computer Methods in Applied Mechanics and Engineering, 193(45-47), 4857-4873.

Noh, H.C. (2005), Stochastic behavior of Mindlin plate with uncertain geometric and material parameters, Probabilistic Engineering Mechanics, 20(4), 296-306.

Noh, H.-C. (2011), Stochastic finite element analysis of composite plates considering spatial randomness of material properties and their correlations, Steel and Composite Structures, 11(2), 115-130.

Phuc, P.M. (2019), Analysis free vibration of the functionally grade material cracked plates with varying thickness using the phase-field theory, Transport and Communications Science Journal, 70(2), 122-131.

Piegl, L.A. and Tiller, W. (1997), The NURBS book, Springer, Berlin; New York.

Rahman, S. and Rao, B.N. (2001), A perturbation method for stochastic meshless analysis in elastostatics, International Journal for Numerical Methods in Engineering, 50(8), 1969-1991.

Real, F.F., Fontanela, F., Ritto, T.G., Batou, A. and Desceliers, C. (2017), A probabilistic model of uncertainties in the substructures and interfaces of a dynamical system: application to the torsional vibration of a drill-string, Archive of Applied Mechanics, 87(4), 685-698.

Shahrokhabadi, S. and Vahedifard, F. (2018), Random Isogeometric Analysis for Modeling Seepage in Unsaturated Soils, Journal of Engineering Mechanics, 144(11), 04018105.

Shang, S. and Yun, G.J. (2013), Stochastic finite element with material uncertainties: Implementation in a general purpose simulation program, Finite Elements in Analysis and Design, 64, 65-78.

Shimpi, R.P. and Patel, H.G. (2006), A two variable refined plate theory for orthotropic plate analysis, International Journal of Solids and Structures, 43(22-23), 6783-6799.

Shinozuka, M. and Deodatis, G. (1991), Simulation of Stochastic Processes by Spectral Representation, Applied Mechanics Reviews, 44(4), 191-204.

Shinozuka, M. and Deodatis, G. (1996), Simulation of Multi-Dimensional Gaussian Stochastic Fields by Spectral Representation, Applied Mechanics Reviews, 49(1), 29-53.

Shiota, I. and Miyamoto, Y. (1997), Functionally Graded Materials 1996 Elsevier Science 
Singh, B.N., Lal, A. and Kumar, R. (2008), Nonlinear bending response of laminated composite plates on nonlinear elastic foundation with uncertain system properties, Engineering Structures, 30(4), 1101-1112.

Taheri, A.H. and Hassani, B. (2014), Simultaneous isogeometrical shape and material design of functionally graded structures for optimal eigenfrequencies, Computer Methods in Applied Mechanics and Engineering, 277, 46-80.

Takada, T. (1990), Weighted integral method in stochastic finite element analysis, Probabilistic Engineering Mechanics, 5(3), 146-156.

Talha, M. and Singh, B.N. (2014), Stochastic perturbation-based finite element for buckling statistics of FGM plates with uncertain material properties in thermal environments, Composite Structures, 108, 823-833.

Tran, L.V., Ly, H.A., Lee, J., Wahab, M.A. and Nguyen-Xuan, H. (2015), Vibration analysis of cracked FGM plates using higherorder shear deformation theory and extended isogeometric approach, International Journal of Mechanical Sciences, 96-97, 65-78.

Vanmarcke, E. and Grigoriu, M. (1983), Stochastic Finite Element Analysis of Simple Beams, Journal of Engineering Mechanics, 109(5), 1203-1214.

Vuong, P.M. and Duc, N.D. (2019), Nonlinear Buckling and Postbuckling of a FGM Toroidal Shell Segment Under a Torsional Load in a Thermal Environment Within Reddy's Third-Order Shear Deformation Shell Theory, Mechanics of Composite Materials, 55(4), 467-482.

Wall, F. and Deodatis, G. (1994), Variability Response Functions of Stochastic Plane Stress/Strain Problems, Journal of Engineering Mechanics, 120(9), 1963-1982.

Wang, C., Yu, T., Shao, G., Nguyen, T.-T. and Bui, T.Q. (2019), Shape optimization of structures with cutouts by an efficient approach based on XIGA and chaotic particle swarm optimization, European Journal of Mechanics - A/Solids, 74, 176-187.

Zhang, H. and Shibutani, T. (2019), Development of stochastic isogeometric analysis (SIGA) method for uncertainty in shape, International Journal for Numerical Methods in Engineering, 118(1), 18-37.

Zhu, W.Q. and Wu, W.Q. (1991), A stochastic finite element method for real eigenvalue problems, Probabilistic Engineering Mechanics, 6(3-4), 228-232. 